\title{
Estudo da espessura da regressão como fator prognóstico nos melanomas cutâneos finos
}

\author{
Regression thickness as prognostic factor in thin cutaneous malignant melanomas
}

Luciana Pantaleão'; Mayra Carrijo Rochael ${ }^{2}$

\author{
unitermos \\ Melanoma \\ Regressão neoplásica \\ espontânea \\ Prognóstico \\ Análise da sobrevida
}

\section{resumo}

Introdução: O diagnóstico de melanomas cutâneos finos (MCF) é cada vez mais freqüente. Essas lesões, inicialmente associadas a excelente prognóstico, têm apresentado recidiva ou metástases e, às vezes, óbito. Muitas variáveis têm sido estudadas e, embora nenhuma delas tenha esclarecido tal comportamento, a regressão e seu possível impacto negativo têm merecido atenção. A regressão tardia é referida pelos autores como de maior relevância no prognóstico. Objetivo: Correlacionar a espessura máxima da área de regressão dos MCFs com o tempo de sobrevida livre de doença. Material e método: Estudo retrospectivo de 84 casos de MCF. Foram utilizados os critérios de Kang et al. (18) para identificação e classificação evolutiva (recente, intermediária e tardia) da regressão. Resultados: Nos $84 \mathrm{MCF}$ s estudados, regressão (em qualquer fase) foi observada em 70 (83,3\%), sendo 30 casos $(35,7 \%)$ com regressão tardia. A medida máxima da regressão variou entre 0,16 e 1,53 mm. O tempo de sobrevida livre de doença variou entre 17 dias e 108 meses. Cinco casos (5,9\%) evoluíram de forma desfavorável, dos quais três eram melanomas in situ (MIS). Não houve correlação entre as variáveis estudadas $(p>0,05)$. Discussão: $O$ significado da regressão sobre o comportamento de MCF é controverso, provavelmente devido a diferentes metodologias utilizadas nos poucos estudos realizados sobre o tema e à grande variabilidade no tamanho e na composição das amostras. Não há consenso na literatura sobre um sistema padronizado de medição da regressão, o que explica, em parte, os resultados controversos obtidos até o momento. Conclusão: Não ficou demonstrada relação entre a espessura da regressão nos melanomas finos e o tempo de sobrevida livre de doença. Estudos futuros, com amostra maior, poderão contribuir para a elucidação da natureza deste fenômeno.

\section{abstract}

Background: The diagnosis of thin cutaneous malignant melanomas has been increasingly more frequent. These lesions, initially associated with excellent prognosis, have presented recurrence or metastases and sometimes have been fatal. Many variables have been studied and, although none of them has explained this behaviour, regression and its possible negative impact have been focused on recently. According to some authors, late regression bears major relevance in the prognosis. Objective: To correlate the maximum regression thickness of thin cutaneous malignant melanomas with disease-free survival time. Materials and method: Retrospective study of 84 cases of thin cutaneous malignant melanomas. The criteria of Kang et al. (1993) for identification and evolutionary classification (early, intermediate and late) of the regression were applied. Results: Regression (in any phase) was observed in 70 out of 84 thin cutaneous malignant melanomas (83.3\%), and 30 cases (35.7\%) showed late regression. The maximum regression thickness measurement ranged from 0.16 to $1.53 \mathrm{~mm}$. Disease-free survival time ranged from 17 days to 108 months. Five cases (5.9\%) had an unfavorable outcome, from which three were in situ melanomas. There was no correlation between the studied variables ( $p>0.05$ ). Discussion: The meaning of regression in thin cutaneous malignant melanomas is controversial, probably due to the different methods applied in the few studies carried out about the subject and the wide variety of size and composition of the samples. There is no medical consensus as to a standardized regression measurement system, which partially explains the controversial results obtained to date. Conclusions: There was no statistical correlation between regression thickness in thick cutaneous melanomas and disease-free survival time ( $(\mathrm{p}$ 0.05). Future studies with wider samples may contribute to a better understanding of this phenomenon. key words

Melanoma

Spontaneous neoplastic regression

Prognosis

Disease-free survival analysis

1. Médica patologista do Hospital Escola Luiz Giuseffe Januzzi; professora assistente da Faculdade de Medicina de Valença (FMV); mestranda em Patologia pela Universidade Federal Fluminense (UFF). 2. Professora adjunta do Departamento de Patologia da UFF, dermatopatologista; doutora em Patologia.

Trabalho realizado no Departamento de Patologia do Hospital Universitário Antônio Pedro (HUAP) da UFF e apresentado como dissertação de mestrado em Patologia em fevereiro de 2007 no HUAP/UFF. 


\section{Introdução}

Nas últimas décadas, o diagnóstico de melanomas cutâneos finos (MCF) (espessura de até $1 \mathrm{~mm}$ ) tem sido cada vez mais freqüente ${ }^{(22)}$ devido ao desenvolvimento de técnicas de detecção clínica precoce e a campanhas de conscientização pública, levando ao aumento do interesse pelas lesões iniciais e suas precursoras. A expectativa de bom prognóstico para essas lesões tem sido abalada porque vários autores relataram recidiva ou metástase em MCF, alguns com curso letal. A freqüência de MCFs com evolução desfavorável varia entre $3 \%$ e $18 \%(10,16,17,28,37,38)$. Apesar de a espessura tumoral ser o principal fator prognóstico para os melanomas em geral, está claro que, para as lesões finas, é necessário identificar parâmetros adicionais que possam predizer o seu comportamento biológico ${ }^{(6,10,13,15,17,19,24,25,28)}$. Um dos parâmetros pesquisados é a regressão.

A regressão, descrita em melanomas pela primeira vez na literatura por Smith e Stehlin ${ }^{(34)}$ em 1965, é a redução do volume tumoral pela resposta imunológica do hospedeiro e tem sido relatada em diversas entidades como nevo melanocítico (nevo halo) e carcinoma basocelular ${ }^{(11,25)}$, sendo freqüente nos melanomas ${ }^{(4,8,25)}$.

Clinicamente, a regressão de melanoma se expressa por diminuição total ou parcial do seu volume, despigmentação, divisão da lesão original em múltiplas lesões menores, depressão ou atrofia no local ${ }^{(25,29)}$.

Os principais critérios adotados para o reconhecimento histológico da regressão são:

- infiltrado de células mononucleares relacionado diretamente com as células tumorais, podendo infiltrar a epiderme ${ }^{(6,13,14,18,25,34) \text {. }}$

- células tumorais apoptóticas são reconhecíveis na fase inicial do processo ${ }^{(14,18)}$;

- redução ou ausência do número de células

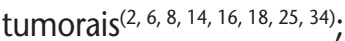

- fibroplasia da derme papilar com disposição do tecido fibroso em camadas horizontais, dando aspecto semelhante a cicatriz ${ }^{(6,13,14,18,25,34)}$;

- angiogênese e telangiectasia( ${ }^{(6,13,14,18,25,34) \text {; }}$

- melanófagos em quantidade variável(2, 6, 8, 14, 16, 18, 25, 34);

- retificação dos cones interpapilares da epiderme (fase

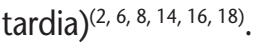

Em 1993, Kang et al. ${ }^{(18)}$ estabeleceram critérios para classificar histologicamente a regressão em recente ou ativa, intermediária e tardia (Tabela 1) ${ }^{(18)}$. Embora o infiltrado brisk faça parte do quadro da regressão recente, são necessários outros elementos (células apoptóticas) para se fazer este diagnóstico. Atualmente há consenso crescente em definir restritivamente a regressão como a fase tardia do processo, por ser a de maior relevância prognóstica ${ }^{(8,24)}$.
Tabela 1

\begin{tabular}{|c|c|c|c|}
\hline & Regressão precoce ou ativa & Regressão intermediária & Regressão tardia \\
\hline Infiltrado linfocitário & $\begin{array}{l}\text { Denso, permeia ou } \\
\text { substitui as células } \\
\text { tumorais }\end{array}$ & $\begin{array}{l}\text { Redução na intensidade do } \\
\text { infiltrado }\end{array}$ & Esparso ou ausente \\
\hline $\begin{array}{l}\text { Células tumorais } \\
\text { degeneradas }\end{array}$ & Presentes, demonstráveis & Em geral, não-demonstráveis & Em geral, não-demonstráveis \\
\hline $\begin{array}{l}\text { Diminuição do } \\
\text { número de células } \\
\text { tumorais }\end{array}$ & Discreta & $\begin{array}{l}\text { Presente, reconhecível pela } \\
\text { perda da continuidade do tumor } \\
\text { em derme, epiderme, ou ambas }\end{array}$ & $\begin{array}{l}\text { Marcada redução ou ausência } \\
\text { de células tumorais }\end{array}$ \\
\hline $\begin{array}{l}\text { Fibrose na } \\
\text { derme papilar }\end{array}$ & Discreta & $\begin{array}{l}\text { Aumento do tecido fibroso } \\
\text { quando comparado com a derme } \\
\text { papilar normal }\end{array}$ & $\begin{array}{l}\text { Tecido conjuntivo denso, } \\
\text { em camadas paralelas à } \\
\text { epiderme, substituindo e } \\
\text { expandindo a derme papilar }\end{array}$ \\
\hline Vasos & Normais & $\begin{array}{l}\text { Neovascularização e } \\
\text { telangiectasia variáveis }\end{array}$ & $\begin{array}{l}\text { Neovascularização e } \\
\text { telangiectasia variáveis }\end{array}$ \\
\hline Melanófagos & $\begin{array}{l}\text { Presentes em número } \\
\text { variável }\end{array}$ & Presentes em número variável & Presentes em número variável \\
\hline $\begin{array}{l}\text { Retificação dos cones } \\
\text { interpapilares da } \\
\text { epiderme }\end{array}$ & Ausente & Pode estar presente & $\begin{array}{l}\text { Presente, pode haver atrofia } \\
\text { da epiderme }\end{array}$ \\
\hline
\end{tabular}

Características da regressão de acordo com a evolução temporal ${ }^{(18)}$

Regressão precoce ou ativa Regressão intermediária

Redução na intensidade do

substitui as células

Presentes, demonstráveis tumo quando comparado com a derme

eovascularização

Presentes em número variável

da epiderme 
Elder et al. ${ }^{(8)}$ consideram apenas aquelas áreas onde há fibroplasia associada a neovascularização, melanófagos e linfócitos, com melanoma presente em um ou ambos os lados, mas não na área de regressão(8)

A prevalência de regressão varia de acordo com a definição de regressão e espessura dos melanomas relatados, sendo mais freqüente nos $\mathrm{MCF}^{(4,16)}$. Estimase que a regressão ocorra em $10 \%$ a $35 \%$ de todos os melanomas e em $31 \%$ a $58 \%$ dos $\mathrm{MCF}^{(4,10,27)}$. Raramente vista antes dos 20 anos de idade, acomete adultos ou idosos, é mais comum no sexo masculino, em tronco e membros inferiores e nos melanomas em fase de crescimento radial| ${ }^{(1,4,6,8,25,27)}$.

O significado da regressão no melanoma sobre o prognóstico do paciente não está estabelecido. Alguns estudos mostraram que MCFs com regressão representam um grupo com alto risco de metástases ${ }^{(4,7,13,14,26,28)}$,enquanto outros não demonstraram relação entre estes fatores ${ }^{(12,19 \text {, }}$ 20, 21, 27, 32). Também tem sido sugerido que tal influência negativa seria mais relevante entre os MCFs do que entre os melanomas espessos, sendo a regressão relacionada com alguns casos de metástases em melanomas in situ $(\mathrm{MIS})^{(6,12,14)}$.

Ronan et al. ${ }^{(30)}$ estudaram 30 casos de MCF com regressão, entre os quais todos aqueles que evoluíram com metástases tinham mais de $75 \%$ de sua área ocupada por alterações regressivas.

Em seu estudo sobre MCFs letais, Slingluff et al. ${ }^{(33)}$ mostraram que a presença de regressão acentuada está associada a significativa redução do tempo de sobrevida livre de doença e que, para tais pacientes, o prognóstico é semelhante ao daqueles com lesões mais espessas.

Posteriormente, Guitart et al. ${ }^{(14)}$ avaliaram 43 casos de MCF com metástases, identificando regressão em 21 casos (49\%). Em 18 (42\%) a regressão ocupava mais de 50\% da superfície da lesão. Tais valores foram significativamente diferentes dos achados em MCFs sem metástases, em que a regressão foi identificada em $19 \%$ dos casos; desses, apenas 5\% apresentavam regressão em mais de 50\% de sua superfície.

Massi et al. ${ }^{(23)}$ demonstraram que, para MCF com regressão, a medida da espessura de Breslow somada à espessura da área de regressão abaixo da última célula tumoral $(T+R)$ é fator prognóstico mais fidedigno do que a medida apenas da espessura tumoral $(T)$.

Entre os estudos que não encontraram relação entre a presença de regressão e a evolução desfavorável nos MCFs, destaca-se o de Leiter et al. ${ }^{(20)}$, que estudaram 12.728 casos de MCFs invasores.

\section{Material e método}

Estudo descritivo, retrospectivo, de peças cirúrgicas de pacientes com MCFs diagnosticados no Serviço de Anatomia Patológica do Hospital Universitário Antônio Pedro, da Universidade Federal Fluminense (HUAP/UFF) e na Divisão de Patologia do Instituto Nacional de Câncer do Rio de Janeiro (INCA) entre os anos de 1990 e 2000, com acompanhamento mínimo de cinco anos.

\section{Critérios de exclusão}

- Pacientes cujo acompanhamento foi perdido ou cujo material foi considerado inadequado ou insuficiente para avaliação;

- pacientes que foram a óbito em menos de cinco anos sem evidência de melanoma ou por causa desconhecida;

- pacientes com segundo melanoma primário de grau maior, com metástase ou recidiva;

- paciente portador de xeroderma pigmentoso.

\section{Processamento do material}

Os cortes em hematoxilina e eosina (HE) do material selecionado foram encaminhados a processamento de rotina para lesões melanocíticas. Além disso, nos casos cujo bloco estava disponível, foram realizadas coloração para fibras elásticas (Weigert) e imuno-histoquímica com anticorpo anti-Melan A, usando-se o cromógeno AEC (marca sigma, código A5754), que confere coloração avermelhada à imunorreação.

\section{Análise do material}

A regressão, quando presente, foi classificada em recente, intermediária e antiga, de acordo com os critérios estabelecidos por Kang et al.(18) (Figuras. 1, 2 e 3) (Tabela 1). Utilizaram-se os termos "focal" para os casos nos quais a regressão ocupou menos de $40 \%$ da área da lesão; "completa" para aqueles em que a regressão ocupou 100\% da área da lesão; e "segmentar" para os demais casos. A medida da espessura máxima da área de regressão foi 
feita desde a célula mais superficial da camada granulosa até o final da fibrose, independente da presença de células tumorais no local. Nos casos com regressão recente, utilizou-se a célula inflamatória mais profunda. Foram relatados, para cada caso, os demais indicadores histopatológicos de prognóstico. O comportamento biológico dos melanomas foi avaliado pelo estudo dos prontuários para acompanhamento e evolução dos pacientes.

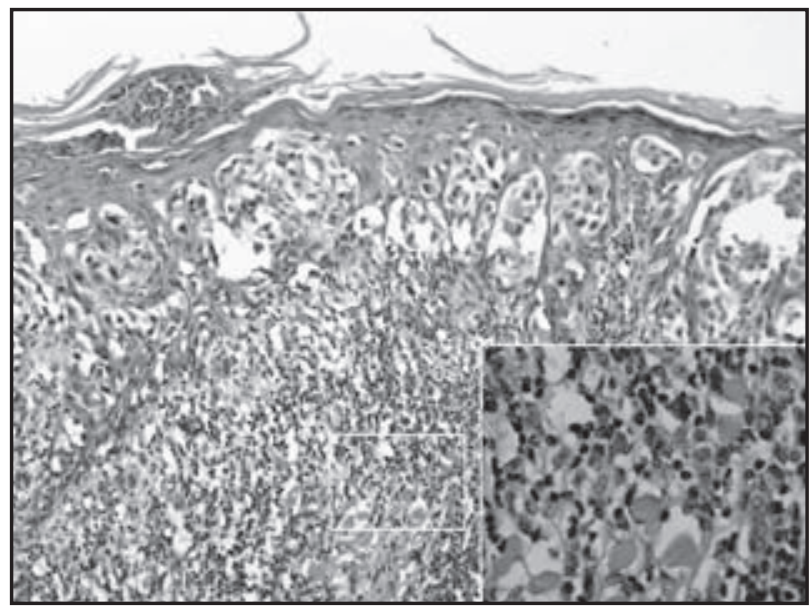

Figura 1 - Melanoma in situ com regressão na fase inicial caracterizado por denso infiltrado inflamatório obscurecendo a periferia do tumor e contendo corpos apoptóticos (no detalhe). Caso 35, HE, 100x (aumento original)

HE: hematoxilina e eosina.

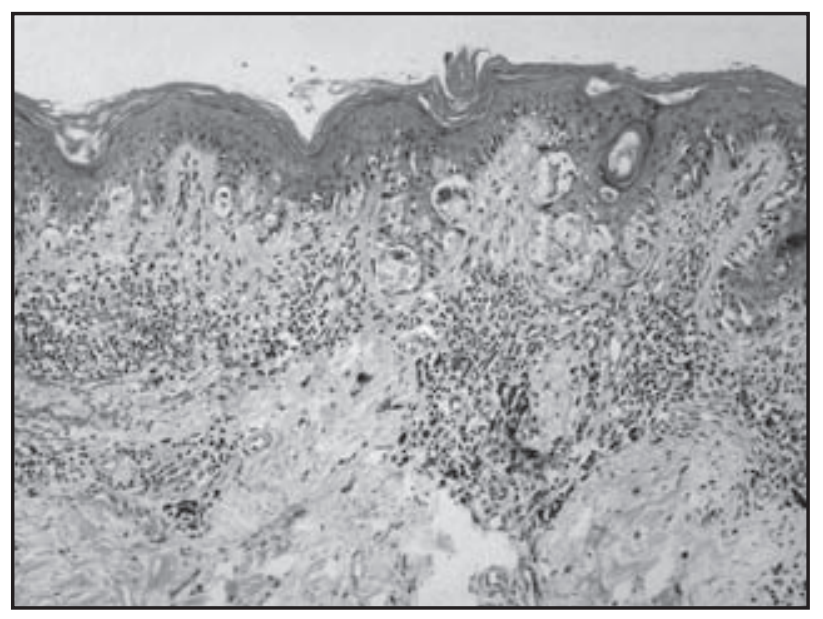

Figura 2 - Área de regressão intermediária em um melanoma in situ. Moderado infiltrado inflamatório, discreta fibrose na derme papilar, melanófagos e proliferação vascular. Caso 1, HE, 100x (aumento original)

HE: hematoxilina e eosina.

\section{Análise estatística}

A relação entre variáveis foi avaliada pelo coeficiente de correlação de Spearman $\left(r_{s}\right)$, quando os critérios de normalidade não se verificaram, e pelo coeficiente de correlação de Pearson ( $r$ ), em caso contrário.

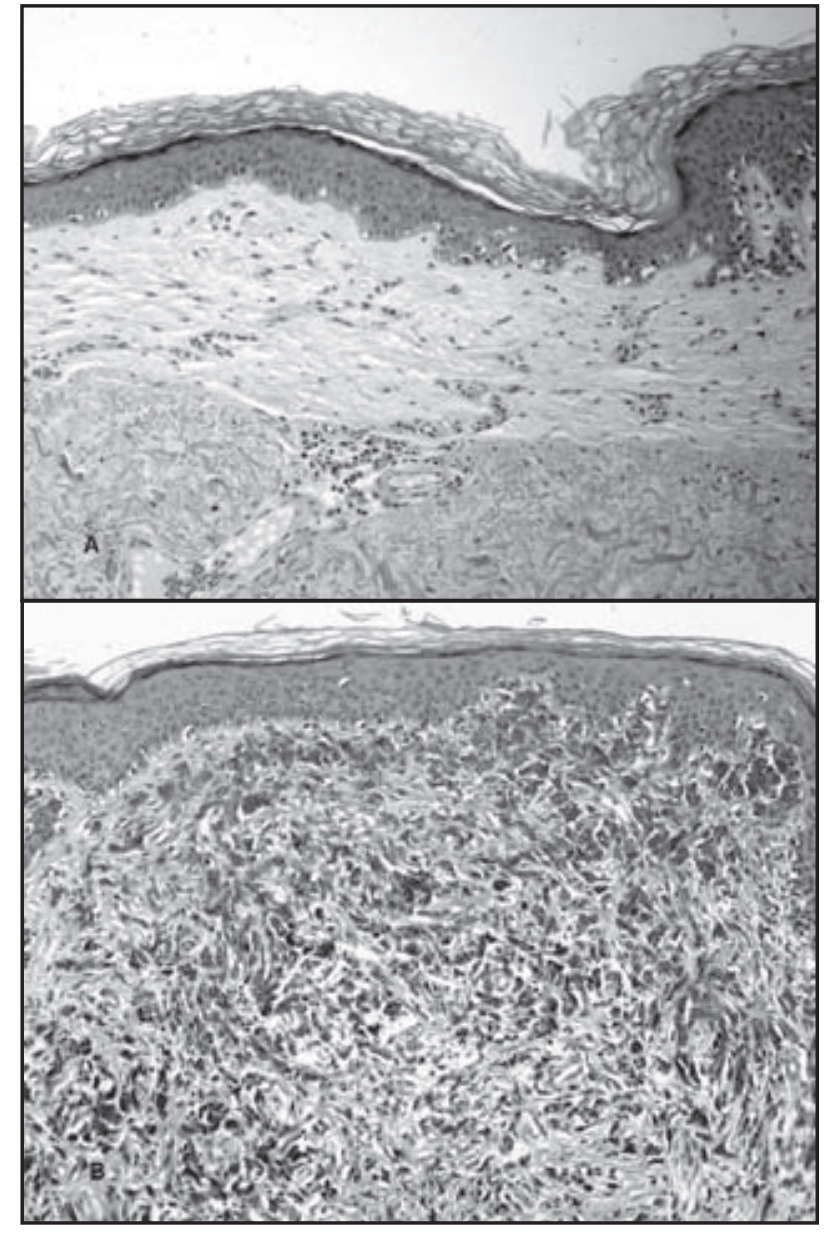

Figura 3 - Regressão tardia caracterizada por: A - Rarefação de células neoplásicas na epiderme e ausência na derme, marcada fibrose em disposição horizontal, vasos neoformados e esparso infiltrado inflamatório. Caso 41, HE, $100 x$ (aumento original). $B$ - Fibrose, proliferação vascular e acentuada diminuição das células neoplásicas na epiderme associadas à presença de acúmulo nodular de melanófagos na derme (melanose). Caso 25, HE, 100x (aumento original)

HE: hematoxilina e eosina.

Utilizou-se o modelo de regressão de Cox para estimar o tempo de sobrevida em função dos fatores prognósticos.

\section{Resultados}

Nossa casuística incluiu 48 mulheres $(57,1 \%)$ e 36 homens $(42,9 \%)$ com idade variando entre 19 e 80 anos (média $=53,5$ ). A faixa etária mais acometida foi entre 50 e 69 anos, tendo as mulheres maior número de casos na sexta década de vida e os homens, na sétima. Na maior parte dos casos, a lesão se localizou no tronco $(30,9 \%)$, seguido pelos membros superiores (29,7\%).

O tipo histológico de maior freqüência foi o disseminativo superficial $(77,3 \%)$. Observamos ainda quatro casos de melanoma lentiginoso acral $(4,7 \%)$ e 15 (18\%) 
de lentigo maligno. Não foram incluídos casos de melanoma nodular.

A distribuição dos 84 casos de melanomas finos em relação aos níveis de Clark foi a seguinte: nível I: 28 casos; nível II: 26; nível III: 24; nível IV: 6; nível V: nenhum caso.

Regressão foi observada em 70 dos 84 casos de melanomas finos, correspondendo a $83,3 \%$ do total, sendo que oito $(9,5 \%)$ casos apresentaram regressão predominantemente na fase recente ou ativa, $32(38,1 \%)$ na fase intermediária e $30(35,7 \%)$ apresentaram regressão antiga.

\section{Distribuição da regressão em relação à idade}

A maior freqüência de regressão foi observada nos pacientes entre 40 e 59 anos de idade, independente da sua classificação. Não observamos regressão no caso de melanoma em paciente com menos de 20 anos e não foi vista regressão antiga em pacientes com menos de 30 anos de idade.

\section{Medida da regressão}

A medida da espessura máxima da regressão nos 70 casos de melanomas finos com regressão variou entre $0,16 \mathrm{~mm}$ e $1,53 \mathrm{~mm}$.

A espessura máxima (Breslow) dos 52 casos de melanomas finos invasores variou entre $0,07 \mathrm{~mm}$ e $1 \mathrm{~mm}$ (média: $0,56 \mathrm{~mm})$.

\section{Sobrevida dos pacientes}

O tempo de sobrevida livre de doença dos 84 pacientes variou entre 17 dias e 108 meses (sobrevida média:
75,2 meses). A curva do tempo de sobrevida livre de doença pelo método de Kaplan-Meier encontra-se na Figura 4.

Cinco casos evoluíram de forma desfavorável, apresentando recidiva local e/ou metástases. Um resumo dos achados clinicopatológicos e da evolução desses cinco casos encontra-se na Tabela 2.

\section{Análise estatística}

O coeficiente de correlação de Spearman indicou, nos 70 casos, inexistência de correlação entre as duas variáveis (espessura máxima da área de regressão, independente da sua fase evolutiva, e tempo de sobrevida livre de doença): $r_{s}=-0,091(p>0,05)$.

Se considerarmos os casos de regressão tardia, também não houve evidência de correlação entre as duas medidas $r_{s}=-0,011(p>0,05)$.

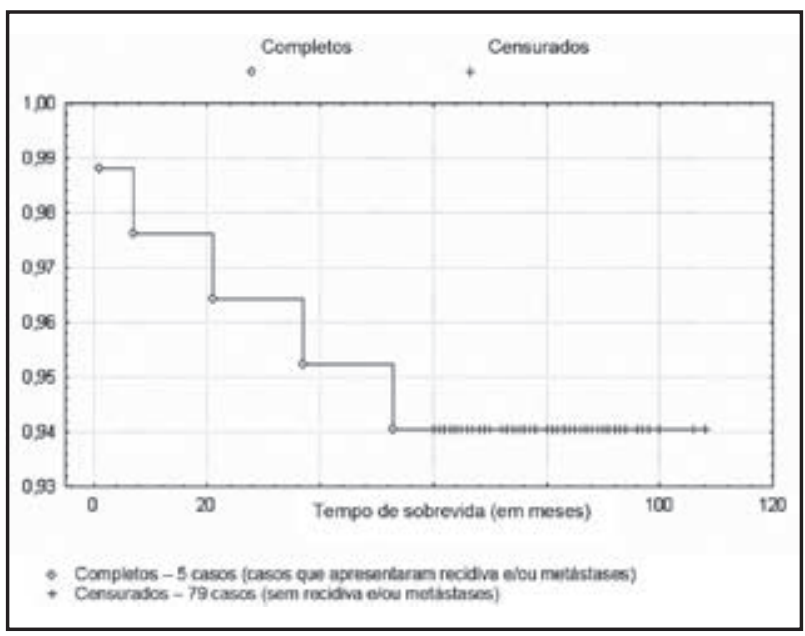

Figura 4 - Curva de sobrevida em meses de 84 pacientes com melanoma fino

\section{Tabela 2 Resumo dos achados dos casos com evolução desfavorável}

\begin{tabular}{lcccccccc}
\hline Caso & TH & Local & NC & EB & Regressão & MR & Sobrevida & Evolução \\
33 & DS & C\&P & I & N/A & Antiga & 1,03 & 37 meses & Recidiva \\
43 & DS & Tronco & IV & 0,75 & Intermediária & 0,82 & 53 meses & $\begin{array}{c}\text { Metástase regional } \\
\text { Recidiva e metástase } \\
\text { regional }\end{array}$ \\
73 & LA & MMII & I & N/A & Ausente & N/A & 7 meses & $\begin{array}{c}\text { Recidiva } \\
80\end{array}$ \\
\hline 6 & DS & C\&P & I & N/A & Antiga & 0,41 & 21 meses & $\begin{array}{c}\text { Metástases } \\
\text { disseminadas } \\
\text { e óbito }\end{array}$
\end{tabular}

TH: tipo histológico; NC: nível de Clark; EB: espessura de Breslow; MR: medida da regressão; DS: disseminativo superficial; C\&P: cabeça e pescoço; N/A: não se aplica; LA: lentiginoso acral; MMII: membros inferiores; MMSS: membros superiores. 


\section{Discussão}

Observamos maior incidência de melanomas finos no sexo feminino, com pico entre 50 e 69 anos, tendo as mulheres sua maior incidência na sexta década de vida e os homens, sétima. Tais valores foram estatisticamente significativos $(p<0,05)$ e coincidem com os da literatura ${ }^{(1,8)}$.

\section{Técnicas utilizadas}

O uso de coloração especial para fibras elásticas (Weigert) para delimitação da área de regressão mostrou-se útil, evidenciando notável diferença entre a área de colágeno neoformado e a derme adjacente, uma vez que a primeira não apresentou fibras elásticas (Figura 5). Essa técnica, já utilizada por outros autores como auxiliar na abordagem de lesões cicatriciais( $^{(9)}$, mostrou-se útil na identificação da área correspondente à regressão.

\section{Regressão}

Considerando-se todas as fases evolutivas do fenômeno, a freqüência de regressão observada nesta amostra $(83,3 \%)$ coincide com dados da literatura ${ }^{(4,10,}$ 27). Seguindo tendências recentes de se considerar apenas a regressão tardia, nossos dados $(35,7 \%)$ também combinam com os da literatura, que indicam regressão em aproximadamente $31 \%$ a $58 \%$ dos $\mathrm{MCF}^{(4,16)}$. A

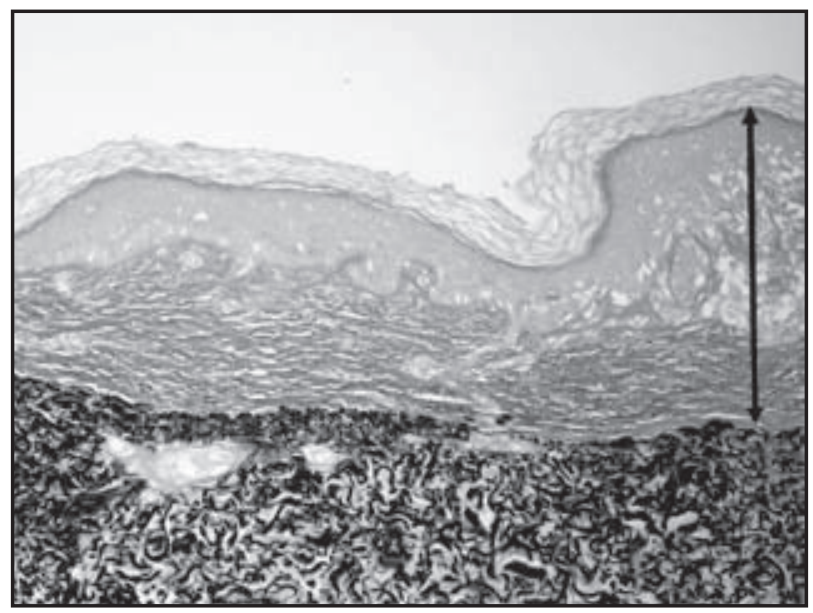

Figura 5 - Melanoma in situ com regressão antiga mostrando a escassez de fibras elásticas na área correspondente à regressão, que foi medida do topo da camada granulosa até o final da fibrose. Caso 41, Weigert, 100x (aumento original) maior freqüência de regressão foi observada nos pacientes entre 40 e 59 anos. Não se observou regressão no paciente com 19 anos de idade.

\section{Regressão como fator prognóstico}

A regressão espontânea representa a resposta imunológica do hospedeiro contra a neoplasia ${ }^{(8,29,31,34,36)}$. A partir desse conceito, seria coerente esperar melhor evolução nos pacientes que apresentam tal reação. Entretanto, em alguns casos, a evolução tem sido contrária a essa expectativa. Uma das possíveis explicações para tal evolução é a disseminação nodal ou visceral que pode ter ocorrido antes do desenvolvimento da regressão, com escape das células disseminadas do processo regressivo da lesão primária( ${ }^{(2)}$. Outra hipótese é a de que melanomas finos, antes da sua redução pela regressão, possam ter sido mais espessos, significando maior gravidade para o paciente ${ }^{(10)}$. Há ainda a possibilidade de que a regressão selecionaria clones celulares mais agressivos e não-responsivos à reação do hospedeiro(4).

Alguns autores mostraram aparente associação entre presença de regressão espontânea e comportamento biológico mais agressivo, com grande divergência nos resultados ${ }^{(12,19,20,21,27,32)}$. O que pode explicar tal divergência são as diferentes metodologias utilizadas. Os critérios para identificar histopatologicamente a regressão variam grandemente entre os autores, dificultando a reprodutibilidade dos resultados. Da mesma maneira, não há padronização em relação aos termos utilizados para se referir à proporção da lesão acometida por fenômenos regressivos quando se empregam os termos "focal" e "parcial". Apenas em relação à regressão completa existe uniformidade no

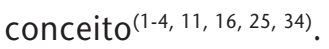

Nos resultados dos trabalhos relatados também encontramos variações nos critérios fundamentais para o estudo do prognóstico dos pacientes, entre eles: a) definição de "melanoma fino" adotada, que variou de $\leq 0,76 \mathrm{~mm}$, segundo Paladugu et al. ${ }^{(28)}$ e Gromet et al. ${ }^{(13)}$, até $\leq 1,5 \mathrm{~mm}$, de acordo com Massi et al. ${ }^{(23)}$; b) tempo de acompanhamento, que variou de um ano, conforme relato de Gromet et al. ${ }^{(13)}$, a 10 anos, segundo Slingluff et al. ${ }^{(33)}$; c) níveis de Clark - alguns autores excluíram os casos de melanomas in situ, como Sondengaard et al. ${ }^{(35)}$ e Massi et al. ${ }^{(23)}$. Nossa amostragem incluiu um caso de MIS com metástase, achado já descrito por outros autores ${ }^{(10,14,33)}$. Por 
isso, ao contrário de Sondengaard et al. ${ }^{(35)}$ e Massi et al. ${ }^{(23)}$, consideramos que incluir tais lesões no estudo pode contribuir para o entendimento de tal fenômeno paradoxal.

\section{Quantificação da regressão}

A variabilidade dos protocolos sugeridos para a quantificação da regressão nos melanomas dificulta a adoção de um sistema de medição padronizado que possa ser aplicado à prática diagnóstica.

O método mais simples foi proposto por Guitart et al. ${ }^{(14)}$ em 2002, quando dividiram os MCFs com regressão em dois grupos: aqueles em que a regressão ocupava menos de $50 \%$ da área da lesão e aqueles em que a regressão preenchia área maior ou igual a 50\% da lesão. Tal método semiquantitativo é fácil de ser incorporado à rotina do patologista. Entretanto não leva em conta a característica peculiar dos melanomas que apresentam comportamento biológico relacionado principalmente com a espessura da lesão(5).

Ronan et al. (30) utilizaram sistema de digitalização de imagens para estimar o percentual da área da lesão acometida por alterações regressivas. Apesar de ser um método preciso de avaliação da área, é de pouca aplicabilidade prática e dificilmente poderá ser incorporado à rotina diagnóstica.

Sondengaard e Hou-Jensen ${ }^{(35)}$ foram os primeiros a propor a utilização da medida em milímetros, tanto da extensão horizontal máxima quanto da espessura máxima da área de fibrose, a partir do topo da camada granulosa, como parâmetros quantitativos da área de regressão. A utilização da lente ocular graduada para avaliação morfométrica dos melanomas já está incorporada à prática do patologista. Tais medidas são precisas e de fácil aplicação, desde que o patologista disponha do corte central no maior eixo da lesão, o que nem sempre é possível, principalmente para os que trabalham com pacientes referidos por outros serviços, onde a lâmina é recebida pronta para consulta do diagnóstico.

O método proposto por Massi et al.(23) foi a medida da espessura da regressão abaixo do tumor (R), no ponto em que foi feita a medida da espessura tumoral $(T)$, somando-se os dois valores $(T+R)$ para serem utilizados como parâmetro prognóstico. Foram estudados 287 pacientes com melanomas invasores e espessura $\leq 1,5 \mathrm{~mm}$, dos quais 32 evoluíram com metástases. Os autores concluíram que a presença de infiltrado inflamatório peri e intratumoral e a espessura da regressão abaixo do tumor $(T+R)$ seriam os principais preditores do prognóstico em MCF. Nesse mesmo trabalho foram testados marcadores morfométricos que levavam em consideração a espessura total da pele (S), expressando os valores em percentual da espessura da pele acometida pelo tumor $(T / S)$ ou pelo tumor e pela regressão $(T+R / S)$. Entretanto, para essas duas novas variáveis, os autores não encontraram relação com o prognóstico ${ }^{(23)}$. Acreditamos que uma limitação desse método seria a possibilidade de haver, em cortes adicionais, áreas mais profundas de regressão em outras localizações que não aquela considerada a maior espessura tumoral, onde foi realizada a medida do índice de Breslow.

\section{Melanomas finos com recidiva e/ou metástases}

Nesta série, apenas cinco casos evoluíram de forma desfavorável, correspondendo a cerca de 5,9\% do total, semelhante aos resultados encontrados pela maioria dos autores $(7,10,16,17,19,26,28,33,37,38)$.

Analisando a ocorrência de regressão nos cinco pacientes que apresentaram evolução desfavorável, três correspondiam a MIS - 10,7\% dos 28 MIS identificados entre os $84 \mathrm{MCF}$, e dois correspondiam a melanomas invasores. Destacamos o caso 73, de MIS sem regressão, que apresentou recidiva local e metástase regional. Guitart et al. ${ }^{(14)}$ relataram caso semelhante e, sem conseguirem explicar tal fenômeno, consideraram a possibilidade de outro melanoma primário oculto, visceral. Hughes e Pritchard(17) atribuíram a ocorrência de metástases, nos casos de MIS, a possível artefato na representação histopatológica. Eles destacaram que, no seu protocolo, o cirurgião responsável pelo tratamento definitivo desempenhou papel crucial realizando dermatoscopia da lesão e marcando, antes da retirada da lesão, as áreas de maior espessura para orientar a clivagem, enfatizando a importância da troca de informações entre o cirurgião e o patologista. No nosso estudo, grande parte dos casos refere-se a pacientes encaminhados ao INCA por outros serviços de diversas partes do país, com processos desiguais de fixação e clivagem. Assim, não se pode excluir a possibilidade de que os cortes da peça tenham sido obtidos, inadvertidamente, de locais que excluíram áreas de invasão do tumor. Por esse motivo 
não é possível, no nosso estudo, atribuir evolução desfavorável em melanomas in situ à regressão completa do componente dérmico do tumor.

No presente estudo, a avaliação estatística dos 70 casos com regressão não mostrou associação entre a espessura máxima da área de regressão e o tempo de sobrevida livre de doença, tanto para regressão em qualquer das suas fases quanto para regressão predominantemente tardia. Desses, apenas quatro casos apresentaram recidiva ou metástase. Faz-se necessária amostra mais representativa de melanomas finos para estudos futuros.

\section{Conclusão}

Depois do estudo de 84 casos de MCF, concluímos que não ficou demonstrada relação entre a espessura da regressão e o tempo de sobrevida livre de doença. Estudos adicionais, com maior número de casos de melanomas finos, são necessários para melhor compreensão do fenômeno.

\section{Referências}

1. BARNHILL, R. L. Malignant melanoma. In: BARNHILL, R. L. et al. Pathology of melanocytic nevi and malignant melanoma. New York: Springer, 2004. p. 238-356.

2. BARR, R. J.; WHITE, G. M.; KIAO, S. Y. Tumoral melanophagocytosis: a rare and confusing pattern of regressed melanoma. J Cutan Pathol, v. 17, p. 287, 1990.

3. BARR, R. J. The many faces of completely regressed malignant melanoma. Pathology, v. 2, p. 359-70, 1994.

4. BLESSING, K; MCLAREN, K. M. Histological regression in primary cutaneous melanomas: recognition, prevalence and significance. Histopathology, v. 20, p. 315-22, 1992.

5. BRESLOW, A. Thickness, cross-sectional areas and depth of invasion in the prognosis of cutaneous melanoma. Ann Surg, v. 172, p. 902-8, 1970.

6. COCHRAN, A. J. et al. Melanocytic tumors: a guide to diagnosis. Philadelphia: Lippincott-Raven, 1997. p. 421.

7. COOK, M. G. et al. Identification of histological features associated with metastatic potential in thin $(<1 \mathrm{~mm})$ cutaneous melanoma with metastases. A study on behalf of the EORTC Melanoma Group. J Pathol, v. 197, p. 188-93, 2002.

8. ELDER, D. et al. Benign pigmented lesions and malignant melanoma. In: ELDER, D. et al. Lever's histopathology of the skin. 9. ed. Philadelphia: Lippincot Williams \& Wilkins, 2004. p. 715-804.

9. ELSTON, D. M. et al. Elastic tissue in scars and alopecia. J Cutan Pathol, v. 27, p. 147-52, 2000.

10. FEARFIELD, L. A. et al. Clinico-patological features of relapsing very thin melanoma. Clin Exp Dermatol, v. 26, n. 8, p. 686-98, 2001.
11. FLAX, S. H. et al. Nodular melanosis due to epithelial neoplasms: a finding not restricted to regressed melanomas. Am J Dermatopathol, v. 20, n. 2, p. 118 22, 1998.

12. FONTAINE, D. et al. Partial regression of primary cutaneous melanoma. Is there an association with sub-clinical sentinel lymph node metastasis? Am J Dermatopathol, v. 25 , n. 5 , p. 371-6, 2003.

13. GROMET, M. A.; EPSTEIN, W. L.; BLOIS, M. S. The regressing thin malignant melanoma: a distinctive lesion with metastatic potential. Cancer, v. 42, n. 5, p. 2282 92, 1978.

14. GUITART, J. etal. Histological characteristics of metastasizing thin melanomas: a case-control study of 43 cases. Arch Dermatol, v. 138, p. 603-7, 2002.

15. HALPERN, A. C.; MARGHOOB, A. A. Thin melanoma: still "excelent prognosis" disease? J Clin Oncology, v. 22, n. 18, p. 3651-3, 2004.

16. HIGH, W. A. et al. Completely regressed primary cutaneous malignant melanoma with nodal and/or visceral metastases: a report of 5 cases and assesment of the litarature and diagnostic criteria. J Am Acad Dermatol, v. 53, n. 1, p. 89-100, 2005.

17. HUGHES, L. E.; PRITCHARD, G. A. Patientes presenting with thin melanomas (less than $0.76 \mathrm{~mm}$ thick) who subsequently have a poor prognosis by development of metastases. Ann Surg, v. 210, p. 249-50, 1998.

18. KANG, S. etal. Histologic regression in malignant melanoma: an interobserver concordance study. J Cutan Pathol, v. 20, p. 126-9, 1993.

19. LEFEVRE, M. et al. Relevance of vertical growth pattern in thin level II cutaneous superficial spreading melanomas. Am J Surg Pathology, v. 27, n. 6, p. 717-24, 2003. 
20. LEITER, U. et al. Prognostic factors of thin cutaneous melanoma: an analysis of the central malignant melanoma registry of the German Dermatological Society. J Clin Oncology, v. 22, n. 18, p. 3660-7, 2004.

21. LISZKAY, G. et al. Relationship between sentinel lymph node status and regression of primary malignant melanoma. Melanoma Res, v. 15, n. 6, p. 509-13, 2005.

22. LUKE, C. G. et al. A critical analysis of reasons for improved survival from invasive cutaneous melanoma. Cancer Causes Control, v. 14, p. 871-8, 2003.

23. MASSI, D. et al. Thin cutaneous malignant melanoma: identification of risk factors indicative of progression. Cancer, v. 85, n. 5, p. 1067-76, 1999.

24. MASSI, D.; FRANCHI, M.; SANTUCCI, M. Prognostic factors in thin cutaneous malignant melanoma. Pathologica, v. 94, p. 282-9, 2002.

25. MASSI, G. et al. Regressing and regressed melanoma. In: MASSI, G. et al. Histologic diagnosis of nevi and melanoma. Würzburg: Springer, 2004, p. 617-31.

26. MANSSON-BRAHME, E. et al. Prognostic factors in thin cutaneous malignant melanoma. Cancer, v. 73, n. 9, p. 2324-32, 1994.

27. MCGOVERN, V. J.; SHAW, H. M.; MILTON, G. W. Prognosis in patients with thin malignant melanoma: influence of regression. Histopathology, v. 7, p. 673-80, 1983.

28. PALADUGU, R. R.; YONEMOTO, R. H. Biologic behavior of thin malignant melanomas with regressive changes. Arch Surg, v. 118, p. 41-4, 1983.

29. PIEPKORN, M.; BARNHILL, R. L. Prognostic factors in cutaneous malignant melanoma. In: BARNHILL, R. L. et al. Pathology of melanocytic nevi and malignant melanoma. New York: Springer, 2004. p. 372-94.
30. RONAN, S. G. et al. Thin malignant melanomas with regression and metastases. Arch Dermatol, v. 123, p. 1326-30, 1987.

31. SALEH, F. et al. Direct evidence on the immune-mediated spontaneous regression of human cancer: an incentive for pharmaceutical companies to develop a novel anti-cancer vaccine. Curr Pharm Des, v. 11, n. 27, p. 3531-43, 2005.

32. SHAW, H. M. et al. Cutaneous melanomas exhibiting unusual biologic behavior. World J Surg, v. 16, p. 196202, 1992.

33. SLIGNLUFF, C. L. et al. Lethal "thin" malignant melanoma: identifying patients at risk. Ann Surg, v. 208, n. 2, p. 150-61, 1988.

34. SMITH Jr., J. L.; STEHLIN Jr., J. L. Spontaneous regression of primary malignant melanomas with regional metastases. Cancer, v. 18, p. 1399-415, 1965.

35. SONDENGAARD, K. HOU-JENSEN, K. Partial regression in thin primary cutaneous malignant melanomas clinical stage I: a study of 486 cases. Virchows Archiv A, v. 408, p. 241-7, 1985.

36. TEFANY, F. J. et al. Immunocytochemical analysis of the cellular infiltrate in primary regressing and non-regressing malignant melanoma. J Invest Dermatol, v. 97, n. 2, p. 197-203, 1991

37. VILMER, C. et al. Thin melanomas with unusual aggressive behavior: a report of nine cases. J Am Acad Dermatol, v. 34, n. 3, p. 439-44, 1996.

38. WOODS, J. E.; SOULE, E. H.; CREAGAN, E. T. Metastasis and death in patients with thin melanomas (less than 0.76 mm). Ann Surg, v. 198, p. 63-4, 1983. 